\title{
Mucin phenotype of differentiated early gastric cancer: an immunohistochemistry study supporting therapeutic decision making
}

This article was published in the following Dove Press journal: Cancer Management and Research

\author{
Elisabetta Cavalcanti' \\ Francesco De Michele' \\ Giulio Lantone ${ }^{2}$ \\ Alba Panarese ${ }^{3}$ \\ Maria Lucia Caruso' \\ 'Histopathology Unit, National Institute \\ of Gastroenterology "S. de Bellis," \\ Research Hospital, Castellana Grotte, \\ Bari, Italy; ${ }^{2}$ Surgery Unit, National \\ Institute of Gastroenterology "S. de \\ Bellis", Research Hospital, Castellana \\ Grotte, Bari, Italy; ${ }^{3}$ Gastroenterology and \\ Endoscopy Unit, National Institute of \\ Gastroenterology "S. de Bellis", Research \\ Hospital, Castellana Grotte, Bari, Italy
}

\begin{abstract}
Introduction: Endoscopic submucosal dissection is widely employed in early gastric cancer (EGC). Foveolar phenotypes should be distinguished from the other differentiated EGC (DEGC) types because of their increased malignant potential. The phenotypic classification could be useful not only for investigating EGC tumorigenesis but also for evaluating the tumor aggressiveness to guide treatment decision making.
\end{abstract}

Methods: On surgical tissue specimens, we studied the mucin phenotype of EGC to distinguish cases with a worse prognosis dictating different therapeutic options or a very close surveillance program. DEGC in our series were classified as mucin foveolar $(51 \%)$ or mucin intestinal (49\%) phenotype. We evaluated correlations among foveolar and intestinal phenotypic markers, tumor patterns, clinicopathologic features and prognostic and therapeutic implications. Immunohistochemistry (IHC) for MUC5AC and CDX2 was performed on 63 EGC patient specimens. MUCA5C was employed as gastric foveolar phenotypic marker and CDX2 as intestinal phenotypic marker.

Results: Foveolar DEGC was significantly associated with larger tumor size $(p=0.01)$, high grade (G2-G3) $(p=0.001)$, vessel permeation $(p=0.05)$, lymph node metastasis $(p=0.001)$ and ulceration $(p=0.001)$, whereas intestinal type DEGC was associated with low grade $(p=0.001)$. Conclusion: IHC determination of the mucin phenotype is an easy, inexpensive method that can provide useful, sensitive markers distinguishing the foveolar or intestinal phenotype in DEGC. The precise identification of the foveolar type, featuring a poorer prognosis, should sound a warning bell mandating very close study of the lesion before endoscopic treatment or contraindicating endoscopic resection in favor of the open surgery option.

Keywords: DEGC, MUC5AC, CDX2, ESD

\section{Introduction}

Most patients with early gastric cancer can now hope for a better quality of life thanks to the recent advent of widespread minimally invasive surgical procedures including endoscopic mucosal resection and submucosal dissection. Early gastric cancer is an invasive carcinoma but it infiltrates no more deeply than the submucosa, regardless of lymph node metastases and tumor size. Many EGC cases have been identified in Japan and well described by Nakamura, ${ }^{1}$ thanks to the very active screening of patients in this geographic area at high risk for gastric cancer. It is essential to identify early stage gastric carcinomas, and target the incipient phases of invasion and metastasis, because early detection and adequate surgical or endoscopic treatment yield a better outcome. The importance of the accurate staging of 
gastric tumors has become even more apparent since the new developments in endoscopic resection techniques. ${ }^{2-4}$ Selected cases of EGC, which respond to specific endoscopic and pathological criteria, are to be considered susceptible to endoscopic treatment with radical intent, rather than open surgery. Therapeutic decision making for endoscopic versus open surgery resection of early gastric carcinoma (EGC) relies upon the clinicopathological findings indicating the risk of lymph nodes metastases (LNM). In EGC with little or no risk of LNM, endoscopic resection, especially endoscopic submucosal dissection (ESD), has become the standard treatment because it features minor patients trauma, less costs and has a higher safety margin, with maximal preservation of gastric function and an excellent long-term prognosis, comparable to that of open surgical resection. ${ }^{5,6}$ For these reasons, ESD is now contemplated as first-line treatment of early gastric cancer in Japanese guidelines. ${ }^{7}$ The lesions to be considered for endoscopic resection, being at very low risk of lymph node metastasis, are the following: a) noninvasive neoplasia (dysplasia) independently of size; b) intramucosal differentiated-type adenocarcinoma, without ulceration (size $\leq 2 \mathrm{~cm}$ absolute indication, $>2 \mathrm{~cm}$ expanded indication); $\mathrm{c}$ ) intramucosal differentiated-type adenocarcinoma, with ulceration, size $\leq 3 \mathrm{~cm}$ (expanded indication); d) intramucosal undifferentiated-type adenocarcinoma, size $\leq 2 \mathrm{~cm}$ (expanded indication); e) differentiated-type adenocarcinoma with superficial submucosal invasion $(\mathrm{sm} 1 \leq 500$ $\mu \mathrm{m}$ ), and size $\leq 3 \mathrm{~cm}$ (expanded indication). Therefore, morphological classification of the EGC subtype is essential for prognostication and treatment decision making. Histologically, gastric carcinomas (GC) are classified under two main types, the intestinal and the diffuse type, depending on whether there are glandular structures, as described by Lauren, ${ }^{8}$ corresponding to the differentiated and undifferentiated types described by Nakamura et al. ${ }^{1}$ This classification, although dated, is still widespread due to its immediacy and simplicity but is proving inadequate for recent chemotherapeutic and surgical approaches. The prognostic relevance of Laurén's classification is still controversial. In some studies, Laurén's histological subtypes of gastric cancer did not show a correlation with the patient's outcome, ${ }^{28}$ whereas other studies demonstrated a prognostic significance for the classification system ${ }^{29}$ other works even demonstrated that Laurén's classification can be used as an independent prognostic factor. ${ }^{30}$ Although Lauren classification gives important information in clinical practice, it is not decisive for prognosis prediction or determining treatment.In particular, the Lauren classification does not distinguish the intestinal or gastric origin in the intestinal/differentiated tumor type. Recent advances in mucin immunohistochemistry have shown that differentiated type tumors may be subclassified into three distinct groups based on their mucin phenotype: foveolar, intestinal and combined. ${ }^{9,10}$ In literature, the incidence of differentiated ECG foveolar-phenotype (DEGC) appears to be $7.9 \%-23.9 \% .{ }^{11}$ Gastric-type mucin phenotypes should be distinguished from the other DEGC types because of their increased malignant potential in the incipient phases of invasion and metastasis. ${ }^{12}$ Early foveolar-phenotype differentiated adenocarcinomas tend to be significantly larger tumors and exhibit higher rates of submucosal invasion than the intestinal-phenotype. ${ }^{13}$ Koseki et $\mathrm{al}^{14}$ reported a significantly higher incidence of lymphatic invasion, venous invasion and lymph node metastasis in gastric-type compared with intestinal-type EGC. Therefore, it is important to correctly identify differentiated gastric adenocarcinomas at an early stage and to discriminate the intestinal or foveolar phenotype of these DEGC. Several studies have reported that a gastric mucin phenotype indicates a poor prognosis, whereas other studies have reported the intestinal mucin phenotype to be associated with a worse outcome, or else no correlation of intestinal or gastric phenotype with survival. ${ }^{15-17}$ Controversies and conflicting results on the prognostic role of Muc5AC expression associated with the foveolar phenotype and cell origin in gastric cancer have ensued. CDX2 is expressed at the early stage of gastric carcinogenesis in intestinal phenotypic elements and could be associated with a suppressed expansion of malignant cells. ${ }^{18,19}$ Furthermore, CDX2 is expressed in the very early stage of gastric carcinogenesis in association with a shift from the gastric to the intestinal phenotypic expression. ${ }^{20}$ This appears to occur at an earlier stage in differentiated cancers compared to undifferentiated forms and may be linked to this suppression of malignant cells expansion. Cdx2-positive GCs had a significantly better outcome than Cdx2-negative GCs. Loss of CDX2 may signal tumor progression in cases of early gastric cancer. ${ }^{21,22}$ Therefore, phenotypic classification may be useful not only for investigating the tumorigenesis of early gastric carcinoma but also for evaluating the tumor aggressiveness and supporting surgical decision making. Moreover, cases of differentiated type carcinoma with a pronounced trend toward invasion and metastasis, even in the early stage, have been observed. In these cases, endoscopic resection is 
not advised due to the likelihood of local recurrence or metastasis. In order to explain this unexpected biological behavior, immunohistochemical evaluations have acquired greater relevance. In this regard, in our study we performed an IHC evaluation of foveolar gastric mucin (MUC5AC) and of an ontogenetic antigen (CDX2), that is highly important for DEGC subclassification, according to the differentiation and development of intestinal cells. Surgically resected tumor specimens from 63 patients with EGC were examined, performing immuno-staining to reveal the gastric foveolar or intestinal origin of the tumor cells. In addition, correlations among foveolar and intestinal phenotypic marker expression, tumor patterns, clinicopathologic features and prognostic and therapeutic implications were evaluated.

\section{Patients and methods}

\section{Patients characteristics}

Paraffin-embedded specimens of 63 EGC tissues were collected from January 2006 to March 2018 at the IRCCS 'Saverio de Bellis' of Castellana Grotte (Ba, Italy). All experiments were performed in accordance with ethical standards, the Declaration of Helsinki, and according to national and international guidelines, and have been approved by review board IRCCS Giovanni Paolo II " Bari (Italy). Written informed consent was obtained from patients. The following clinicopathological characteristics were collected for all patients: age, gender, macroscopic features, histological type, tumor grade, presence of ulceration, vessel permeation, perineural infiltration and lymph node metastasis.

\section{Pathological assessment}

Histology was assessed in all tumors by two pathologists who reviewed FFPE tissue sections stained with hematoxylin and eosin (H\&E), and a representative paraffin block from each specimen was chosen for IHC analysis. On $\mathrm{H} \& \mathrm{E}$ and PAS mucin-stained sections, the histological characteristics of cells, tumor grade, the presence of ulcerations, perineural infiltration, vessel permeation and the presence of lymph node metastasis were evaluated. The specimens were classified according to both European Society of Gastrointestinal Endoscopy and AJCC 8th edition (American Joint Committee on Cancer) guidelines. In this regard, in Table 1 the grade refers only to the intestinal phenotype because diffuse type carcinoma is high grade and classified as grade 3.
Table I Clinicopathologic features of 63 patients with early gastric cancer

\begin{tabular}{|c|c|c|}
\hline Features & & $\%$ \\
\hline \multicolumn{3}{|l|}{ Gender } \\
\hline Men & 41 & 65 \\
\hline Women & 22 & 35 \\
\hline \multicolumn{3}{|l|}{ Age, years } \\
\hline Median, range (min-max) & 67.35 & \\
\hline & $(34-91)$ & \\
\hline \multicolumn{3}{|l|}{ Macroscopic features } \\
\hline Type I (protruding) elevated & 16 & 25.40 \\
\hline Type II (superficial) flat & 27 & 42.86 \\
\hline Type III (excavated) depressed & 20 & 31.75 \\
\hline \multicolumn{3}{|l|}{ Istological type } \\
\hline Intestinal & 33 & 52.38 \\
\hline Diffuse & 19 & 30.16 \\
\hline Mixed & 11 & 17.46 \\
\hline \multicolumn{3}{|c|}{$\begin{array}{l}\text { Tumor size }(\mathrm{cm}) \text { median, range (min- } \\
\max )\end{array}$} \\
\hline Intestinal & $2.61(0.5-7)$ & \\
\hline Diffuse & 1.77 & \\
\hline & $(0.5-3.05)$ & \\
\hline \multicolumn{3}{|l|}{ Grading of intestinal type } \\
\hline GI & 13 & 39.39 \\
\hline G2 & 12 & 36.36 \\
\hline G3 & 7 & 21.21 \\
\hline \multicolumn{3}{|l|}{ Vessel permeation } \\
\hline Absent & 39 & 61.90 \\
\hline Present & 24 & 38.10 \\
\hline \multicolumn{3}{|l|}{ Perineural infiltration } \\
\hline Absent & 38 & 60.32 \\
\hline Present & 25 & 39.68 \\
\hline \multicolumn{3}{|l|}{ Lynphonodal metastasis } \\
\hline Absent & 57 & 90.48 \\
\hline Present & 6 & 9.52 \\
\hline
\end{tabular}

\section{IHC evaluation}

$\mathrm{HC}$ analysis for MUC5AC and CDX2 was performed in the FFPE of 63 patients with EGC. Tumor sections of $4 \mu \mathrm{m}$ were freshly cut and dried at $60^{\circ} \mathrm{C}$ for $30 \mathrm{~min}$. IHC analysis was carried out on the sections after deparaffinization for 30 mins and then rehydration in grades of alcohol. Antigen retrieval was performed at $90^{\circ} \mathrm{C}$ for 20 mins with CitratoBuffer. To assess MUC5AC, a foveolar epithelium glycoprotein, and $\mathrm{CDX} 2$, an intestine-specific transcription factor, we employed these antibodies on the EGC tissues (clone 45M1 at 1:100 
dilution; clone CDX2-88 at 1:100 dilution) using an automated autostainer (cat. K5007, Dako, Glostrup, Denmark). The Real Envision DAB Substrate Kit (DAKO) was used according to the manufacturer's instructions. The staining pattern of MUC5AC and CDX2 antibodies was as expected: cellular membranous and cytoplasmic for Muc5AC and only nuclear for CDX2. MUC5AC and CDX2 expression were scored for all staining patterns, according to both the staining intensity and the percentage of positively stained cells, by two independent, blinded pathologists; discrepancies in the interpretation of scoring were resolved by consensus. Using these methods, the DGCs were classified into two phenotypes: gastric phenotype (G-type) and intestinal phenotype (I-type). The criteria for classification of the G-type and I-type mucin phenotypes were as follows: G-type included those in which $20 \%$ of the cells were stained positively for gastric type markers (MUC5AC) and were stained negatively for intestinal markers (CDX2), whereas I-type comprised CDX2 positive cells that were negative for gastric type markers (Figure 1).

\section{Statistical analysis}

Correlations between MUC5AC and CDX2 tissue expression and clinicopathological parameters were determined by Chi-square test and Fisher's exact test. A $p$-value less than $0.05\left(^{*}\right)$ was considered statistically significant. All evaluations were performed using StataCorp. 2007 Stata Statistical
Software: release 10 (StataCorp LP, College Station, TX, USA).

\section{Results}

\section{Patient population}

The clinicopathologic features of the 63 cases of EGC are summarized in Table 1. Patients median age was 67.35 years (range: 34-91): there were 22 females (35\%) and 41 males (65\%). Of the 63 cases of EGC, $33(52.38 \%)$ were intestinal type; there were $19(30.16 \%)$ cases of diffuse type cancers and $11(17.46 \%)$ cases of mixed type cancers. The baseline characteristics of the two types of EGC are described in Table 2. The EGC Intestinal type was significantly larger than the foveolar type $(p=0.01)$. The incidence of perineural infiltration, a known ominous morphological pattern in the oncologic field, was significantly higher in intestinal type compared to diffuse type carcinoma cases $(p=0.02)$. However, there were no significant differences in vessel permeation, lymph node metastasis and ulcerative phenomena between intestinal type and diffuse type carcinomas.

\section{Phenotypic expression of DEGC}

To evaluate the origin and phenotypic expression of DEGC types, we analyzed Muc5AC (revealing foveolar mucin glycoprotein) and CDX2 (revealing intestinal cell
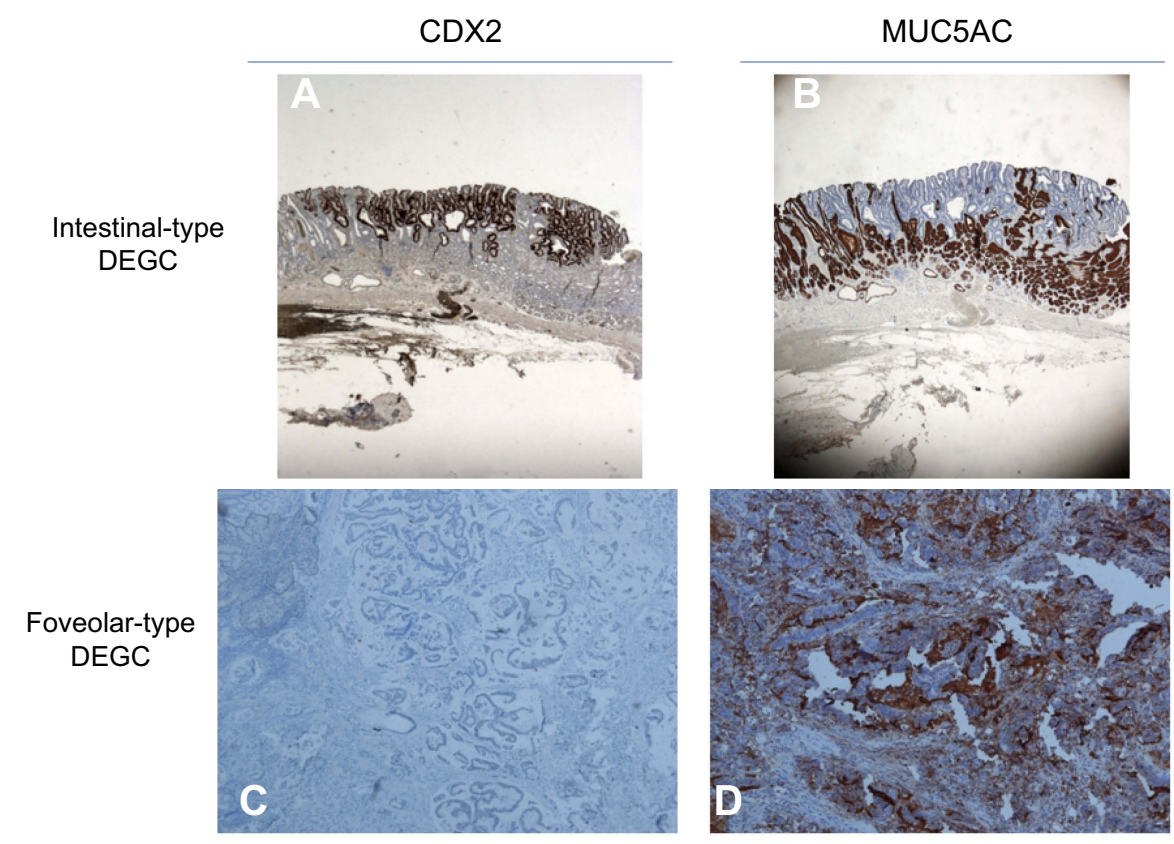

Figure I IHC patterns in type of differentiated EGCs (A and B) intestinal - type CDX2+ MUC5AC $-(\times 20)(\mathbf{C}$ and $\mathbf{D})$ gastric - type CDX2 - MUC5AC+ $(\times 100)$ Abbreviations: DEGC, differentiated EGC; EGC, early gastric cancer; IHC, immunohistochemistry. 
Table 2 Baseline characteristics of the two types of early gastric carcinoma

\begin{tabular}{|c|c|c|c|}
\hline Variables & $\begin{array}{l}\text { Intestinal } \\
\text { type }(n=33)\end{array}$ & $\begin{array}{l}\text { Diffuse } \\
\text { type }(n=19)\end{array}$ & $p$-value* \\
\hline $\begin{array}{l}\text { Tumor size }(\mathrm{cm}) \\
\text { Median, range } \\
(\text { min- max })\end{array}$ & $2.61(0.5-7)$ & $1.77(0.5-3.05)$ & 0.01 \\
\hline $\begin{array}{l}\text { Vessel } \\
\text { permeation } \\
\text { Absent } \\
\text { Present }\end{array}$ & $\begin{array}{l}21(63.6) \\
12(36.4)\end{array}$ & $\begin{array}{l}\text { II (57.9) } \\
8(42.1)\end{array}$ & 0.68 \\
\hline $\begin{array}{c}\text { Perineural } \\
\text { infiltration } \\
\text { Absent } \\
\text { Present }\end{array}$ & $\begin{array}{l}17(51.5) \\
16(48.5)\end{array}$ & $\begin{array}{l}16(84.2) \\
3(15.8)\end{array}$ & $0.035^{\wedge}$ \\
\hline $\begin{array}{l}\text { Lynphonodal } \\
\text { metastasis } \\
\text { Absent } \\
\text { Present }\end{array}$ & $\begin{array}{l}29(87.9) \\
3(9.1)\end{array}$ & $\begin{array}{l}16(84.2) \\
3(15.8)\end{array}$ & $0.66^{\wedge}$ \\
\hline $\begin{array}{l}\text { Ulceration } \\
\text { Absent } \\
\text { Present }\end{array}$ & $\begin{array}{l}12(36.4) \\
21(63.6)\end{array}$ & $\begin{array}{l}5(26.3) \\
13(68.4)\end{array}$ & $0.53^{\wedge}$ \\
\hline
\end{tabular}

Notes: *Chi-square test, ${ }^{\wedge}$ Fisher's exact test.

origin) tissue expression in $33(52.38 \%)$ DEGC. The correlation between Muc5AC or CDX2 expression and gender was not significant $(p=0.07 ; 0.04)$ (Table 3 ). Muc5AC was observed in $17(51 \%)$ of 33 EGC cases. Importantly, Muc5AC expression was absent in all $16 \mathrm{G} 1$ cases $(100 \%)$, while Muc5AC positivity in tumor cell membranes was detected in 9 G2 cases $(100 \%)$ and 8 G3 cases (100\%) (Table 3). On the other hand, Muc5AC positivity was expressed in all $17(100 \%)$ cases presenting ulceration and perineural infiltration $(17 ; 100 \%)$ but only in 13 of 17 cases with vessel permeation (Table 3).

Hence, the expression of MUC5AC was associated with aggressive tumor characteristics, including high grade ( $p=0.001)$, ulceration $(p=0.001)$, vessel permeation $(p=0.001)$ and perineural infiltration $(p=0001)$. By contrast, CDX2 expression was present in $16(49 \%)$ of 33 EGC cases and was found to be significantly associated with low-grade tumors ( $p=0.001)$. All $16(100 \%)$ cases of positive CDX2 expression lacked the above-listed aggressive tumor characteristics (Table 3). On the basis of the combined expression patterns of CDX2 and MUC5AC, the DEGC were classified as intestinal phenotype ( $\mathrm{CDX2}^{+}$, Muc5AC ), 16 cases (49\%), and foveolar phenotype (CDX2 $\left.{ }^{-}, \mathrm{Muc}^{\mathrm{AC}} \mathrm{C}^{+}\right), 17$ cases (51\%) (Figure 1).
Importantly, not only does the foveolar phenotype feature a larger tumor size but also a greater percentage of cases infiltrating the submucosa $(15 ; 88 \%)$, while the intestinal phenotype featured a large percentage of intramucosal cases (12; $75 \%$ ) (Table 4).

Thus, foveolar-phenotype DEGC was associated with a more advanced histological grade (G2-G3), ulceration, that was present in all of these patients, and also a significantly greater vascular involvement.

\section{Discussion}

In the last two decades, endoscopic treatment of EGC with curative intent has been widely performed, adopting well-defined absolute and expanded inclusion criteria. To clarify gastric tumorigenesis it is essential to investigate phenotypic markers expression and correlations between clinicopathological findings in the early tumor phase. Approximately $30 \%$ of DGC express mainly gastric type mucin. ${ }^{23}$ Differentiated foveolar EGC, measuring less than $2 \mathrm{~cm}$ in diameter, are universally recognized as treatable by ESD regardless of histological type. Differentiated gastric phenotype cancer, in the early phase, is prone to transformation to undifferentiated type cancer and to growth with deeper infiltration in the wall layers. This type of DGC should be distinguished from the others because of its tendency to invade and metastasize already during the incipient phase. ${ }^{24}$ The importance of discriminating these phenotypes is also due to the fact that early gastric foveolar-phenotype differentiated adenocarcinomas are more aggressive, tend to be significantly larger tumors and exhibit higher rates of submucosal invasion than intestinal-phenotype EGC. Therefore, to clarify gastric tumorigenesis it is essential to investigate phenotypic markers expression, that is also driven by genetic alterations in the early tumoral phase. In literature, the reported incidence of gastric foveolar-phenotype differentiated adenocarcinoma appears to range from $7.9 \%$ to $23.9 \% .{ }^{11,25} \mathrm{In}$ our series, in the DEGC cases, we found a higher percentage of foveolar phenotype tumors. In the present study, the intestinal origin and foveolar phenotypic mucin glycoprotein expression was examined in 33 (52.38\%) DEGC. On the basis of the combined expression patterns of CDX2 and MUC5AC, the DEGC were classified as $16(49 \%)$ intestinal mucin phenotype $\left(\mathrm{CDX}^{+}, \mathrm{Muc} \mathrm{AC}^{-}\right)$and 17 (51\%) foveolar phenotype (CDX2 ${ }^{-}, \mathrm{Muc} \mathrm{AC}^{+}$) (Figure 1). In particular, foveolar phenotype DEGC were significantly associated with high grade (G2-G3) $(p=0.001)$, large 
Table 3 Phenotypic markers expression in differentiated type early gastric cancer

\begin{tabular}{|c|c|c|c|c|c|c|c|c|}
\hline & \multicolumn{3}{|c|}{ MUC5AC expression } & \multirow[t]{3}{*}{$P$-value } & \multicolumn{3}{|c|}{ CDX2 expression } & \multirow[t]{3}{*}{$P$-value } \\
\hline & Positive & Negative & Total & & Positive & Negative & Total & \\
\hline & n (\%) & n (\%) & n (\%) & & n (\%) & n (\%) & n (\%) & \\
\hline \multicolumn{9}{|l|}{ Gender } \\
\hline Male & $9(47.4)$ & $10(52.6)$ & 19 & $0.58^{*}$ & $9(45,0)$ & II (55) & $20(60)$ & $0.62^{*}$ \\
\hline Female & $8(57.1)$ & $6(42.9)$ & 14 & & $7(53,8)$ & $6(46.1)$ & $13(40)$ & \\
\hline \multicolumn{9}{|l|}{ Histological grade } \\
\hline Grade I & 0 & $16(100)$ & 16 & $<0.001^{\wedge}$ & $16(100)$ & 0 & 16 & $<0.001^{\wedge}$ \\
\hline Grade 2 & $9(100)$ & 0 & 9 & & 0 & $8(100)$ & 8 & \\
\hline Grade 3 & $8(100)$ & 0 & 8 & & 0 & $9(100)$ & 9 & \\
\hline \multicolumn{9}{|l|}{ Ulceration } \\
\hline Absent & 0 & $16(100)$ & 16 & $<0.001^{\wedge}$ & $16(48.4)$ & $17(5 \mid .5)$ & 33 & \\
\hline Present & $17(100)$ & 0 & 17 & & 0 & 0 & 0 & \\
\hline \multicolumn{9}{|l|}{ Vessel permeation } \\
\hline Absent & $4(20.0)$ & $16(80.0)$ & 20 & $<0.001^{\wedge}$ & 16 & 17 & 33 & \\
\hline Present & $13(100)$ & 0 & 13 & & 0 & 0 & 0 & \\
\hline \multicolumn{9}{|l|}{ Perineural infiltration } \\
\hline Absent & 0 & $16(100)$ & 16 & $<0.001^{\wedge}$ & 16 & 17 & 33 & \\
\hline Present & $17(100) 10$ & 0 & 17 & & 0 & 0 & 0 & \\
\hline
\end{tabular}

Notes: *Chi-square test, ' Fisher's exact test.

tumor size $(p=0.01)$, vessel permeation $(p=0.05)$, lymph node metastasis $(p=0.001)$ and ulceration $(p=0.001)$, whereas intestinal phenotype DEGC were significantly associated with low grade $(p=0.001)$ and no aggressive tumor characteristics. Moreover, there were distinct differences in tumor aggressiveness according to the phenotypic marker expressions of early-stage gastric differentiatedtype tumors. Differentiated-type gastric carcinomas with a foveolar phenotype have been suggested to be more likely to transform into undifferentiated-type carcinoma. In literature (14), among early gastric carcinomas, the gastric phenotype is reported to be an independent factor associated with lymph node metastasis. In our 63 cases of EGC, the 17 foveolar mucin phenotype cases, as defined by IHC assay, showed a greater lesion diameter $(2.86$ vs $1.77 \mathrm{~cm})$ and more submucosal involvement $(88 \%$ vs $25 \%$ ) than the intestinal mucin phenotype. Confirming the more aggressive nature of the foveolar phenotype, the loss of CDX2 occurs as a signal of tumor progression from early to advanced GC, marking the shift to gastric phenotype expression. The worldwide diffusion and standardization of endoscopic treatment, the refinements in ESD techniques, instruments and devices defined in the last two decades, together with the extension of the criteria and the conditions for eligibility for less invasive resective procedures must work in parallel with a better knowledge of the biology of this heterogeneous tumor burden, searching for multifocality with synchronous and metachronous lesions. ESD is a highly effective treatment modality for EGC in cases that meet the absolute and expanded criteria described in current guidelines and is also a valid resource to identify untreatable patients, who are often elderly with severe comorbidities and cannot undergo surgery due to the high operation risk. ${ }^{26}$ For these patients, palliative ESD remains the unique opportunity to avoid serious complications such as perforation or hemorrhage or stenosis requiring urgent salvage measures. Patients with gastric mucin phenotype tumors have a poorer prognosis than those with intestinal mucin phenotype, suffering more peritoneal recurrence after curative resection, whereas hematogenous recurrence occurred more frequently in the intestinal mucin phenotype. ${ }^{27}$ As for pedunculated colonic adenomas containing adenocarcinoma, endoscopically treated, also in EGC accurate evaluation of the patterns indicative of greater LNMs risk is the main criterion dictating the treatment choice and later evaluating its successful outcome. Therefore, all possible additional evaluation methods serving to indicate those cases that can safely 
Table 4 Mucine phenotype and histopathological pattern

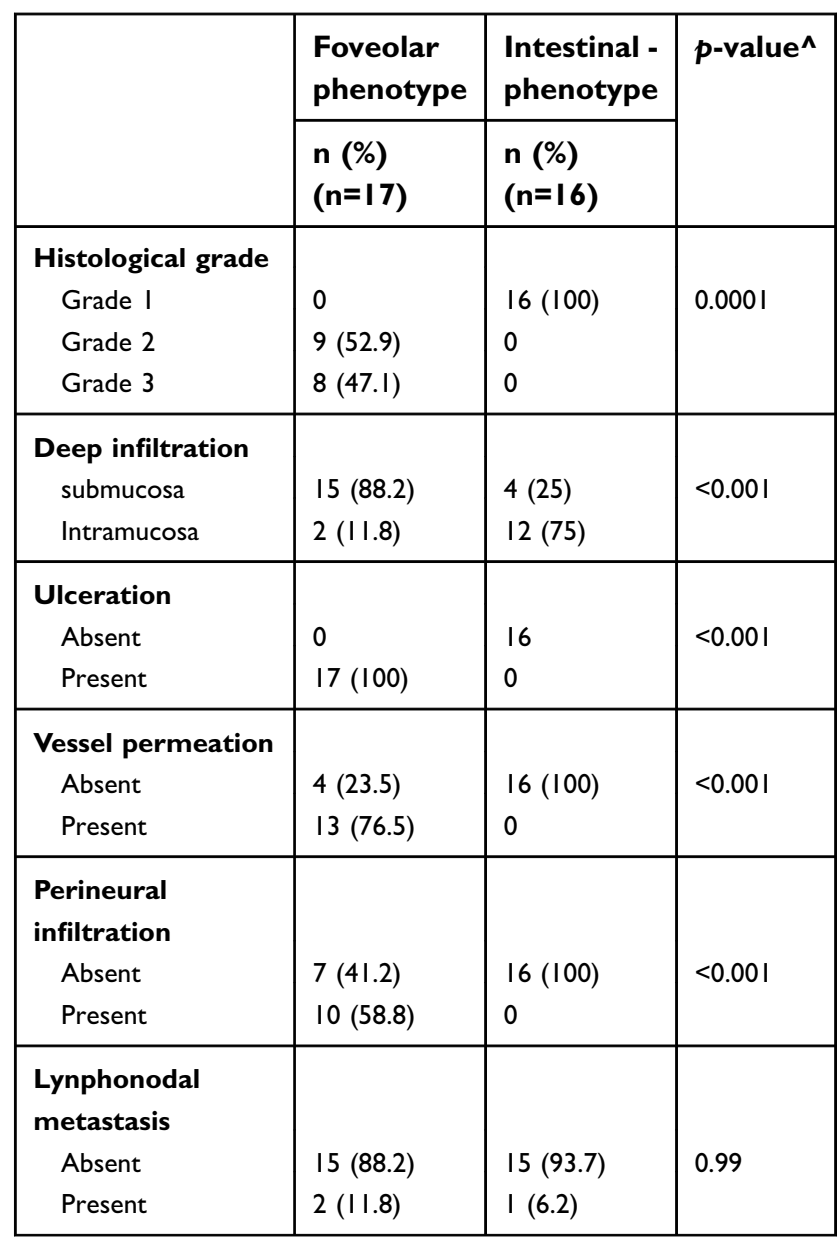

Note: ^Fisher's exact test.

undergo endoscopic resection procedures, with curative intent, ranging from echo-endoscopy to assess the submucosal invasion level, to imaging studies to exclude LNM, must be employed. Our study suggests that IHC evaluation of the mucin phenotype, widely considered to indicate a negative prognosis if of foveolar phenotype, offers a useful support to treatment decision making. This research underlines that the immunohistochemical profile of DEGC mucin expression, as a prognostic factor, should be an integral part of the preoperative phase investigation guiding therapeutic decision making. In conclusion, IHC determination of CDX2 and MUC5AC is an easy, inexpensive method and provides useful sensitive markers discriminating the foveolar from the intestinal phenotype in DEGCs. The identification of foveolar phenotype EGC should ring a warning bell, mandating a very close study of the lesion before endoscopic treatment or contraindicating endoscopic resection procedures, favoring an open surgery approach, in the absence of contraindications, or a very close surveillance program following endoscopic dissection to screen for local recurrence and tumor metastasis elsewhere in the gastric cavity.

\section{Pathological assessment}

Histology was assessed in all tumors by two pathologists who reviewed FFPE tissue sections stained with hematoxylin and eosin (H\&E), and a representative paraffin block from each specimen was chosen for IHC analysis. On H\&E and PAS mucin-stained sections, the histological characteristics of cells, tumor grade, the presence of ulcerations, perineural infiltration, vessel permeation and the presence of lymph node metastasis were evaluated. The specimens were classified according to both European Society of Gastrointestinal Endoscopy and AJCC 8th edition (American Joint Committee on Cancer) guidelines.

\section{Ethical approval}

This article does not contain any studies with human participants or animals performed by any of the authors.

\section{Disclosure}

All of the authors declare no conflicts of interest related to this study.

\section{References}

1. Nakamura K, Sugano H, Takagi K. Carcinoma of the stomach in incipient phase: its histogenesis and histological appearances. Gann. 1968;59:251-258.

2. Tada M, Murakami A, Karita M, Yanai H, Okita K. Endoscopic resection of early gastric cancer. Endoscopy. 1993;25:445-450. doi:10.1055/s-2007-1010365

3. Lambert R. Endoscopic treatment of esophagogastric tumours. Endoscopy. 1996;28:27-37. doi:10.1055/s-2007-1005415

4. Bourke MJ, Neuhaus H, Bergman J. Endoscopic submucosal dissection: indications and application in western endoscopy practice. Gastroenterology. 2018;154(7):1887-1900. doi:10.1053/j. gastro.2018.01.068

5. Kim GH. Endoscopic submucosal dissection for early gastric cancers with uncommon histology. Clin Endosc. 2016;49(5):434-437. doi:10.5946/ce.2016.127

6. Hahn KY, Park CH, Lee YK, et al. Comparative study between endoscopic submucosal dissection and surgery in patients with early gastric cancer. Surg Endosc. 2018;32(1):73-86. doi:10.1007/s00464-017-5640-8

7. Japanese Gastric Cancer Association. Japanese classification of gastric carcinoma: 3rd English edition. Gastric Cancer. 2011;14(2):101-112. doi:10.1007/s10120-011-0041-5

8. Lauren P. The two histological main types of gastric carcinoma: diffuse and so-called intestinal-type carcinoma. An attempt at a histo-clinical classification. Acta Pathol Microbiol Scand. 1965;64:31-49. doi:10.1111/apm.1965.64.1.31

9. Shiroshita H, Watanabe H, Ajioka Y, Watanabe G, Nishikura K, Kitano S. Re-evaluation of mucin phenotypes of gastric minute well-differentiated-type adenocarcinomas using a series of HGM, MUC5AC, MUC6, M-GGMC, MUC2 and CD10 stains. Pathol Int. 2004;54(5):311-321. doi:10.1111/j.1440-1827.2004.01625.x 
10. Kabashima A, Yao T, Sugimachi K, Tsuneyoshi M. Gastric or intestinal phenotypic expression in the carcinomas and background mucosa of multiple early gastric carcinomas. Histopathology. 2000;37:513-522. doi:10.1046/j.1365-2559.2000.01008.x

11. Kabashima A, Yao T, Sugimachi K, Tsuneyoshi M. Relationship between biologic behavior and phenotypic expression in intramucosal gastric carcinomas. Hum Pathol. 2002;33:80-86.

12. Hayakawa M, Nishikura K, Ajioka Y, Aoyagi Y, Terai S. Reevaluation of phenotypic expression in differentiated-type early adenocarcinoma of the stomach. Pathol Int. 2017;67(3):131-140. doi:10.1111/pin.12506

13. Matsuoka M, Aizawa Y, Nagamata H, Sutoh S, Miyagawa Y, Torii A. Significance of the mucin phenotype of early gastric cancer. Jikeikai Med J. 2003;50:29-36.

14. Koseki K, Takizawa T, Koike M, Ito M, Nihei Z, Sugihara K. Distinction of differentiated type early gastric carcinoma with gastric type mucin expression. Cancer. 2000;89:724-732.

15. Kocer B, Soran A, Kiyak G, et al. Prognostic significance of mucin expression in gastric carcinoma. Dig Dis Sci. 2004;49(6):954-64.].

16. Lee HS, Lee HK, Kim HS, Yang HK, Kim YI, Kim WH. MUC1, MUC2, MUC5AC, and MUC6 expressions in gastric carcinomas: their roles as prognostic indicators. Cancer. 2001;92(6):1427-1434. doi:10.1002/(ISSN)1097-0142

17. Saito A, Shimoda T, Nakanishi Y, Ochiai A, Toda G. Histologic heterogeneity and mucin phenotypic expression in early gastric cancer. Pathol Int. 2001;51(3):165-171. doi:10.1046/j.1440-1827.2001.01179.x

18. Silberg DG, Swain GP, Suh ER, Traber PG. Cdx1 and cdx2 expression during intestinal development. Gastroenterology. 2000;119:961-971. doi:10.1053/gast.2000.18142

19. Ha Kim G, Am Song G, Youn Park D, et al. CDX2 expression is increased in gastric cancers with less invasiveness and intestinal mucin phenotype. Scand $J$ Gastroenterol. 2006;41:880-886. doi:10.1080/00365520500497140

20. Mizoshita $\mathrm{T}$, Inada $\mathrm{K}$, Tsukamoto $\mathrm{T}$, et al. Expression of the intestine-specific transcription factors, $\mathrm{Cdx} 1$ and $\mathrm{Cdx} 2$, correlates shift to an intestinal phenotype in gastric cancer cells. J Cancer Res Clin Oncol. 2004;130(1):29-36. doi:10.1007/s00432-003-0503-1
21. Saad RS, Ghorab Z, Khalifa MA, Xu M. CDX2 as a marker for intestinal differentiation: its utility and limitations. World J Gastrointest Surg. 2011;3(11):159-166. doi:10.4240/wjgs.v3.i11.159

22. Liu Q, Teh M, Ito K, Shah N, Ito Y, Yeoh KG. CDX2 expression is progressively decreased in human gastric intestinal metaplasia, dysplasia and cancer. Mod Pathol. 2007;20:1286-1297. doi:10.1038/ modpathol.3800968

23. Tajima Y, Shimoda T, Nakanishi Y, et al. Gastric and intestinal phenotypic marker expression in gastric carcinomas and their prognostic significance: immunohistochemical analysis of 136 lesions. Oncology. 2001;61:212-220. doi:10.1159/000055377

24. Ishikawa $\mathrm{S}$, Togashi $\mathrm{A}$, Inoue $\mathrm{M}$, et al. Indications for EMR/ESD in cases of early gastric cancer: relationship between histological type, depth of wall invasion, and lymph node metastasis. Gastric Cancer. 2007;10(1):35-38. doi:10.1007/s10120-006-0407-2

25. Zou X. Clinicopathology of early gastric carcinoma: an update for pathologists and gastroenterologists. Gastrointest Tumors. 2017;3(3-4):115-124. doi:10.1159/000456005

26. Isomoto $H$, Kurumi $H$. Management of non-curative endoscopic submucosal dissection for early gastric cancer: do we have enough data to support this? Transl Gastroenterol Hepatol. 2017;2:35. doi: $10.21037 / \operatorname{tgh}$

27. Lee OJ, Kim HJ, Kim JR, Watanabe H. The prognostic significance of the mucin phenotype of gastric adenocarcinoma and its relationship with histologic classifications. Oncol Rep. 2009;21(2):387-393.

28. Mönig S, Baldus SE, Collet PH, et al.. Histological grading in gastric cancer by Goseki classification: correlation with histopathological subtypes and prognosis. Anticancer Res. 2001;21(1B):617-20.

29. Yu CC, Levison DA, Dunn JA, et al. Pathological prognostic factors in the second British Stomach Cancer Group trial of adjuvant therapy in resectable gastric cancer. Br J Cancer. 1995;71(5):1106-10.

30. Qiu MZ, Cai MY, Zhang DS, et al. Clinicopathological characteristics and prognostic analysis of Lauren classification in gastric adenocarcinoma in China. J Transl Med. 2013;11:58.

\section{Publish your work in this journal}

Cancer Management and Research is an international, peer-reviewed open access journal focusing on cancer research and the optimal use of preventative and integrated treatment interventions to achieve improved outcomes, enhanced survival and quality of life for the cancer patient.
The manuscript management system is completely online and includes a very quick and fair peer-review system, which is all easy to use. Visit http://www.dovepress.com/testimonials.php to read real quotes from published authors. 